\title{
DESARROLLO INICIAL DEL ROBLE ANDINO EN RESPUESTA AL MANEJO EN VIVERO Y DURANTE LA PLANTACIÓN
}

\author{
Initial development of andean oak in response \\ to management in nursery and at planting
}

\author{
María Claudia Díez ${ }^{1}$ Yira L. Sepúlveda² \& Flavio Moreno³
}

Diez, M.C., Sepúlveda, Y.L. \& Moreno, F. (2017). Desarrollo inicial del roble andino en respuesta al manejo en vivero y durante la plantación. Colombia Forestal, 20(2), 118-130.

Recepción: 18 de marzo 2016

\section{Resumen}

Las plantaciones de Quercus humboldtii se requieren para el desarrollo de programas de restauración de robledales y de reforestación comercial. En este trabajo se estudió el efecto del manejo en vivero (iluminación relativa y fertilización), las características del sitio de plantación (cobertura vegetal) y la fertilización al momento del trasplante, sobre el crecimiento inicial en campo de Q. humboldtii. La cobertura de rastrojo presentó condiciones más favorables sobre el crecimiento en altura, y en ella la fertilización en vivero y durante la plantación generó un efecto positivo sobre la altura y el diámetro, pues las deficiencias nutricionales adquiridas desde el vivero disminuyeron el desarrollo en campo. Las deficiencias en N, P y $\mathrm{Ca}$, fueron más restrictivas que las deficiencias en B, Mg, S y K. La iluminación relativa en vivero no influyó sobre el crecimiento en esta cobertura. Por su parte, en pastizales el crecimiento diamétrico fue mayor; la fertilización (en vivero y campo) no afectó el crecimiento en altura, pero si en diámetro. En pastizales la deficiencia en $\mathrm{N}$ fue la más restrictiva, mientras que las deficiencias en $\mathrm{S}$ y $\mathrm{K}$
Aprobación: 22 de mayo de 2017

fueron las menos restrictivas. Los árboles sometidos a alta iluminación en vivero crecieron más al plantarlos en pastizales.

Palabras clave: árboles tropicales, Fagaceae, fertilización, luz, pastizales, Quercus humboldtii Bonpl., rastrojos altos, silvicultura de plantaciones

\begin{abstract}
The establishment of plantations of Quercus humboldtii is required for the development of oak restoration programs and for commercial reforestation. In this work the effect of nursery management (relative lighting and fertilization), the characteristics of the planting site (vegetation cover) and fertilization at transplant, on the initial growth of $Q$. humboldtii is determined. The results indicate that the vegetation cover of secondary forest presented more favorable conditions for height growth; in this cover fertilization in the nursery and at planting had positive effects on height and diameter. Nutritional deficiencies acquired from the nursery decreased the development in the field. Deficiencies of N, P, and Ca were more restrictive than the deficiencies of $\mathrm{B}, \mathrm{Mg}, \mathrm{S}$, and $\mathrm{K}$. The lighting on nursery did not influence growth in
\end{abstract}

1 Grupo de Investigación en Ecología y Silvicultura de Especies Forestales Tropicales. Departamento de Ciencias Forestales, Facultad de Ciencias Agrarias, Universidad Nacional de Colombia sede Medellín. Medellín, Colombia. mcdiez@unal.edu.co Autor para correspondencia.

2 Grupo de Investigación en Ecología y Silvicultura de Especies Forestales Tropicales, Departamento de Ciencias Forestales, Facultad de Ciencias Agrarias, Universidad Nacional de Colombia sede Medellín. Medellín, Colombia. yiras81@hotmail.com

3 Grupo de Investigación en Ecología y Silvicultura de Especies Forestales Tropicales, Departamento de Ciencias Forestales, Facultad de Ciencias Agrarias, Universidad Nacional de Colombia sede Medellín. Medellín, Colombia. fhmoreno@unal.edu.co 
this plant cover. Meanwhile, in pastures, diameter growth was higher; fertilization (in the nursery and in the field) did not affect the growth of height but in diameter. In pastures, $\mathrm{N}$ deficiency was the most restrictive, while the $S$ and $K$ deficiencies were the least restrictive. Trees that grew under highlighting in nursery grew more when planted in pastures.

Keywords: tropical trees, Fagaceae, fertilization, light, pastures, Quercus humboldtii Bonpl., secondary forests, plantation forestry

\section{INTRODUCCIÓN}

Quercus es uno de los géneros de especies leñosas más abundantes y de mayor importancia económica en el hemisferio norte, con cerca de 500 especies (Nixon, 1993; Manos et al., 1999). Sin embargo, en Suramérica solo se encuentra una especie de este género, el $Q$. humboldtii, que se distribuye en los Andes tropicales de Colombia; de ahí que su nombre común sea roble andino. La reducción dramática de los bosques andinos, sumado a la fuerte extracción selectiva de $Q$. humboldtii para su aprovechamiento en múltiples aplicaciones (Pacheco \& Pinzón, 1997), ha motivado su inclusión como especie forestal amenazada, hasta el punto de ser necesaria una veda nacional para su tala desde hace más una década (Cárdenas \& Salinas, 2006). Además, esta especie es dominante en los "robledales" que ocupan áreas extensas de la zona andina de Colombia entre los 1000 y 3600 $\mathrm{m}$ de altitud, considerados como ecosistemas prioritarios para la conservación por su alta biodiversidad y endemismo (Myers, 2000), así como por los servicios ambientales que se derivan de ellos (Armenteras et al., 2011). Por esto, determinar las condiciones de manejo en vivero que permitan el establecimiento exitoso de plantaciones de roble andino es de gran importancia, tanto para programas de conservación y recomposición de robledales, como para reforestación con fines comerciales en la zona andina.

Las técnicas de producción de material vegetal en vivero influyen en el desempeño de las plantas en campo (Gilman, 1994; Landis, 1985; van den Driessche, 1984). Generalmente, la fertilización en vivero mejora los parámetros morfológicos y funcionales, los cuales aumentan la capacidad de sobrellevar el estrés ambiental durante el primer año de plantación (Villar-Salvador et al., 2004; Andivia et al., 2012). Sin embargo, este efecto varía con la especie y el estrés ambiental en el sitio de la plantación, hasta el punto que en algunas especies esta práctica no es determinante para la sobrevivencia de los árboles en campo, especialmente cuando se plantan en zonas con baja disponibilidad de agua (Trubat et al., 2011).

En un trabajo previo (Sepúlveda et al., 2014), se encontró que el manejo en vivero y, particularmente la aplicación de nutrientes en la forma de fertilizantes, modifica el desarrollo de los árboles en vivero. No obstante, se desconoce si este efecto perdura cuando se plantan en los andisoles degradados por agricultura y ganadería, que son generalmente los sitios disponibles para reforestación en las zonas altas de las cordilleras del país (Tejedor et al., 2012).

El propósito de este estudio fue analizar el efecto de diferentes condiciones de manejo en vivero reportadas en Sepúlveda et al. (2014), sobre el desempeño en el campo de $Q$. humboldtii en plantaciones establecidas bajo dos sistemas: plantación monoespecífica a plena exposición en pastos y plantación de enriquecimiento bajo dosel en rastrojo alto. Específicamente, el estudio se enfocó en responder las siguientes preguntas: 1) ¿afecta el tipo de fertilizante aplicado y el régimen de sombra utilizada en vivero la sobrevivencia y el crecimiento inicial en campo de los árboles de roble en cada uno de estos sistemas de plantación?; 2) ¿la fertilización aplicada al momento de la plantación 
tiene algún efecto sobre el desempeño de la plantación?; y 3) ¿cuál de los dos sistemas de plantación evaluados es mejor para esta especie?

\section{MATERIALES Y MÉTODOS}

\section{Área experimental}

El experimento se estableció en un terreno localizado a 2460 m de altitud, en el municipio de Guarne, Antioquia $\left(6^{\circ} 13^{\prime} 19^{\prime \prime} \mathrm{N}\right.$ y $\left.75^{\circ} 28^{\prime} 41^{\prime \prime} \mathrm{W}\right)$. En él se separaron dos áreas con diferentes coberturas vegetales: cobertura de pastos y cobertura de rastrojo alto, de tal manera que ofreciera condiciones contrastantes de iluminación y se pudieran establecer los siguientes modelos de plantación de roble: monoespecífica a plena exposición y de enriquecimiento de rastrojos. El lote tiene una pendiente promedia de $25 \%$ y los suelos corresponden a depósitos de ceniza volcánica (Andisoles), sobre anfibolitas y serpentinitas, con las características químicas que se muestran en la tabla 1. Las condiciones bioclimáticas del área corresponden a la zona de vida de bosque húmedo Montano Bajo
(bh-MB) (Holdridge, 1996), con precipitación promedia anual de $1815 \mathrm{~mm}$ en régimen bimodal, humedad relativa media anual del aire de $83 \%$ y temperatura media anual de $14.9^{\circ} \mathrm{C}$ (Empresas Públicas de Medellín, 1994).

\section{Establecimiento y monitoreo del ensayo}

Los árboles que se utilizaron para la plantación estuvieron en vivero durante cuatro meses a partir de la germinación con el fin de promover una buena formación de raíces y permitir que con el tiempo se agotasen las reservas de nutrientes de las semiIlas (las cuales tienen un diámetro promedio de 30 a $35 \mathrm{~mm}$, altura de 35 a $40 \mathrm{~mm}$ y una masa seca de 6 a 8 g.). Los árboles crecieron bajo tres condiciones de iluminación relativa (IR): alta (plena exposición), media (30 \% de iluminación relativa) y baja (15\% de iluminación relativa). Estas fueron sometidas a distintos tratamientos de fertilización, comenzando por uno completo (TC) que contenía nitrógeno $(\mathrm{N})$, fósforo $(\mathrm{P})$, potasio $(\mathrm{K})$, calcio $(\mathrm{Ca})$, magnesio $(\mathrm{Mg})$, azufre $(\mathrm{S})$ y boro $(\mathrm{B})$, así como tratamientos en los cuales se eliminó del tratamiento completo sustrayendo algún elemento, uno a uno,

Tabla 1. Valores promedio del análisis químico* de los suelos bajo cobertura de rastrojo y pasto en el lote donde se estableció la plantación de roble $(Q$. humboldtii).

\begin{tabular}{lcc}
\hline Parámetro & $\begin{array}{c}\text { Valores para el suelo en } \\
\text { la cobertura de rastrojo }\end{array}$ & $\begin{array}{c}\text { Valores para el suelo } \\
\text { en la cobertura de pasto }\end{array}$ \\
\hline $\mathbf{p H}$ & 4.70 & 5.60 \\
$\mathbf{M O}(\%)$ & 29.64 & 20.69 \\
$\mathbf{C a}\left(\mathrm{cmol}(+) \mathrm{kg}^{-1}\right)$ & 0.81 & 7.31 \\
$\mathbf{M g}\left(\mathrm{cmol}^{-1}+\mathrm{kg}^{-1}\right)$ & 1.82 & 0.42 \\
$\mathbf{K}\left(\mathrm{cmol}^{-}+\mathrm{kg}^{-1}\right)$ & 0.39 & 0.26 \\
$\mathbf{P}\left(\mathrm{mg} \mathrm{kg}^{-1}\right)$ & 2.60 & 2.90 \\
$\mathbf{A l}\left(\mathrm{cmol}^{(+)} \mathrm{kg}^{-1}\right)$ & 0.60 & - \\
$\mathbf{N}(\%)$ & 0.81 & 0.71 \\
$\mathbf{B}\left(\mathrm{mg} \mathrm{kg}^{-1}\right)$ & 0.41 & 0.42 \\
\hline
\end{tabular}

* Para la determinación analítica se usaron los siguientes métodos: nitrógeno: Micro- Kjedahl; fósforo: ácido L-ascórbico, potasio, calcio y magnesio: absorción atómica. Los análisis se realizaron en el Laboratorio de Suelos y Nutrición Vegetal de la Universidad Nacional de Colombia. Detalles de la preparación de las muestras y los análisis químicos están disponibles en Westerman et al. (1990). Los análisis se realizaron en una muestra compuesta tomada ene 5 puntos de cada lote entre 0 y $30 \mathrm{~cm}$ de profundidad 
de tal forma que se tuvieron los tratamientos $-\mathrm{N}$, $-\mathrm{P},-\mathrm{K},-\mathrm{Ca},-\mathrm{Mg},-\mathrm{S}, \mathrm{y}-\mathrm{B}$, además de un tratamiento testigo (T0) sin fertilización. De esta manera, se tuvieron nueve tratamientos de fertilización (ver Sepúlveda et al., 2014). Antes del traslado al campo, los árboles se llevaron a condiciones de estrés hídrico durante 15 días y al momento de la plantación se realizó una poda del 50 \% del área foliar y liberación de raíces (Cervantes et al., 2001).

Los árboles se plantaron en campo en dos coberturas vegetales con condiciones de iluminación relativa contrastantes: una zona con pasto kikuyo (Pennisetum clandestinum) a plena exposición y un rastrojo alto con especies como roble andino $(Q$. humboldtii), carate (Vismia baccifera), sietecueros (Tibouchina lepidota), entre otras; con altura media entre 6 y 10 m e iluminación relativa en el sotobosque de $32.6 \pm 8.4 \%$. La iluminación relativa se midió como la proporción de la radiación incidente a campo abierto, medida con sensores cuánticos.

En ambos casos los árboles se plantaron en hoyos de $30 \mathrm{~cm}$ de diámetro y $30 \mathrm{~cm}$ de profundidad, a una distancia de $5 \mathrm{~m}$ (en cuadrícula de $5 \times 5 \mathrm{~m}$, para una densidad de 400 árboles $\mathrm{ha}^{-1}$ ). Antes de la plantación se hizo una desyerba manual con machete de $1 \mathrm{~m}$ de diámetro alrededor de cada hoyo. La mitad de los árboles recibieron un tratamiento de fertilización adicional al momento de la plantación que consistió en la aplicación de un fertilizante compuesto N:P:K de grado 10:30:10, a razón de $35 \mathrm{~g}$ al fondo del hoyo. Los árboles se plantaron conservando el sustrato que traían del vivero y, luego, el hoyo se acabó de llenar con el suelo restante de la excavación. Después de un año de establecidos los árboles en campo, se evaluó la sobrevivencia y se calcularon los incrementos anuales de altura y diámetro en el cuello de la raíz.

\section{Diseño experimental y análisis estadístico}

Los árboles de los distintos tratamientos se distribuyeron al azar en las coberturas (pasto y rastrojo alto). En cada una se aplicaron dos niveles de fertilización: sin fertilización en campo y con fertilización en campo; y por cada nivel de fertilización en campo se plantaron árboles provenientes de cada sistema de manejo en vivero con tres repeticiones. En total se utilizaron 324 árboles.

En cada cobertura se evaluó el efecto del manejo en vivero (iluminación relativa y tipo de fertilizante aplicado) y la aplicación o no de fertilizante en campo sobre el desarrollo de los árboles, en un diseño factorial $2 \times 3 \times 9$, que corresponde a dos niveles del factor fertilización en campo, tres niveles del factor iluminación relativa en vivero y nueve niveles del factor tipo de fertilizante aplicado en vivero, detallados en párrafos anteriores (figura 1). Los datos se evaluaron mediante análisis de varianza según el modelo lineal aditivo del diseño experimental. Cuando los análisis de varianza arrojaron diferencias significativas se realizaron pruebas de comparación de medias de Tukey con nivel de significancia del $95 \%$. Las variables utilizadas para los análisis fueron incremento anual en altura $(\mathrm{H})$ y en diámetro del cuello de la raíz (DCR). Para todos los casos se comprobó normalidad de los datos mediante el test de Kolmogorov-Smirnov. Los dos sistemas de plantación de $Q$. humboldtii (monoespecífico a plena exposición y enriquecimiento bajo dosel de rastrojo alto) se compararon mediante pruebas t de Student.

\section{RESULTADOS}

\section{Efecto de las condiciones en vivero y la fertilización en campo sobre la plantación de roble establecida como enriquecimiento de rastrojo}

El manejo en vivero y la fertilización en campo no tuvieron efecto significativo sobre la sobrevivencia de los árboles de roble después de un año en este sistema de plantación; la sobrevivencia promedio fue del $94 \%$. Pero sí tuvieron un efecto significativo sobre el crecimiento de esta plantación de roble tanto en altura como en el diámetro del cuello de la raíz. No fueron significativas para los parámetros 


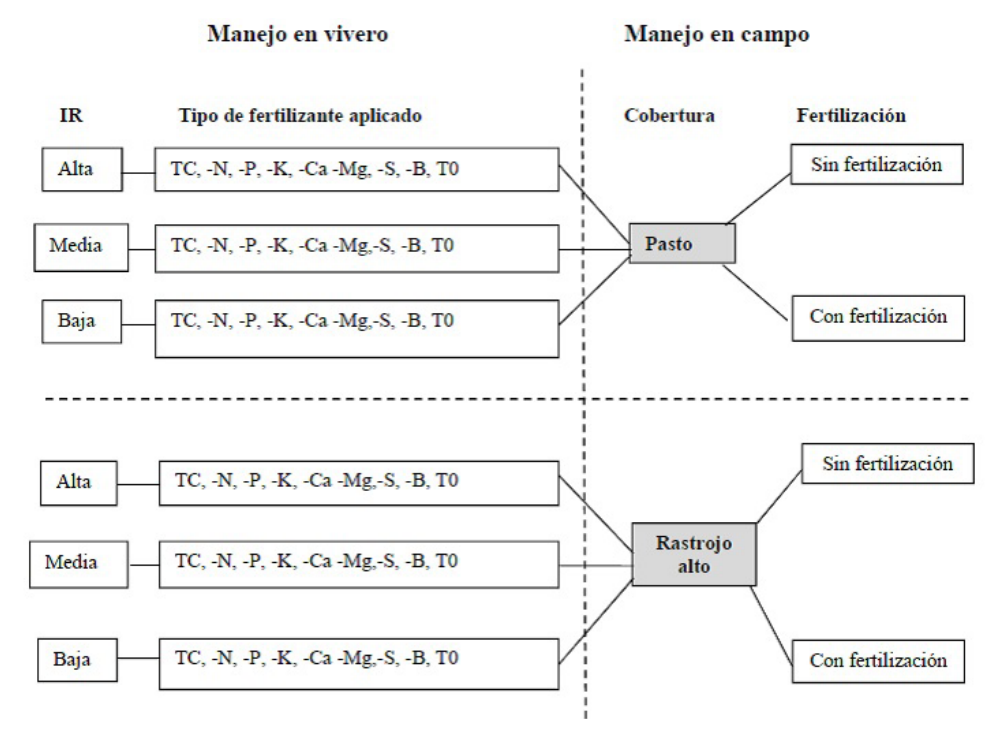

Figura 1. Esquema del diseño experimental que se aplicó en el ensayo de plantación de roble (Q. humboldtii) en las coberturas de rastrojo alto y pastizal. En cada cobertura se plantaron arbolitos con y sin fertilización al momento de la plantación, los cuales provenían de diferentes esquemas de manejo en vivero en cuanto a la iluminación relativa (IR) y tipo de fertilizante aplicado ( $\mathrm{TC}=$ tratamiento completo, $-\mathrm{N}=$ tratamiento completo menos nitrógeno, $-\mathrm{P}=$ tratamiento completo menos fósforo, $-\mathrm{K}=$ tratamiento completo menos potasio, $-\mathrm{Ca}=$ tratamiento completo menos calcio, $-\mathrm{Mg}=$ tratamiento completo menos magnesio, $-\mathrm{S}=$ tratamiento completo menos azufre, $-\mathrm{B}=$ tratamiento completo menos boro, $\mathrm{T} 0=$ tratamiento testigo).

medidos la iluminación relativa en el vivero ni las interacciones entre los factores evaluados (tabla 2).

$\mathrm{Al}$ analizar separadamente el efecto del tipo de fertilizante usado en vivero (figura 2), se observa que, en general, se presentó una tendencia de disminución del crecimento por la aplicación de fertilizantes con elementos faltantes. Específicamente con respecto al incremento anual en altura, las diferencias fueron estadísticamente significativas entre el grupo de mayor crecimiento, conformado por las plantas que recibieron en vivero los tipos de fertilizante completo (TC) y sin potasio $(-K)$ y las que no recibieron fertilización (T0), fertilizante sin nitrógeno (-N) y sin fósforo (-P). El incremento anual en el diámetro del cuello también presentó una tendencia de disminución por la aplicación de fertilizantes con elementos faltantes, pero en este caso las mayores diferencias estadísticas se presentaron entre las plantas que recibieron tratamiento completo en vivero (TC) y las que recibieron

Tabla 2. Valores de $\mathrm{P}$ del efecto de los tratamientos sobre el desarrollo de árboles de roble andino (Q. humboldii) después de un año de su plantación en cobertura de rastrojo alto (efectos significativos en negrilla). FC: fertilización en campo, IRV: iluminación relativa en vivero, FV: tipo de fertilizante aplicado en vivero.

\begin{tabular}{llcccccc}
\hline \multicolumn{1}{c}{ Variable } & \multicolumn{1}{c}{ FC } & \multicolumn{1}{c}{ IRV } & FV & FCxIRV & FCxFV & IRVxFV & FCxIRVxFV \\
\hline $\begin{array}{l}\text { Incremento anual altura } \\
\text { Incremento anual diámetro }\end{array}$ & $0.002^{*}$ & $0.062 \mathrm{~ns}$ & $0.029^{*}$ & $0.141 \mathrm{~ns}$ & $0.964 \mathrm{~ns}$ & $0.264 \mathrm{~ns}$ & $0.073 \mathrm{~ns}$ \\
cuello de la raíz & $0.000^{* * *}$ & $0.096 \mathrm{~ns}$ & $0.000^{* * *}$ & $0.582 \mathrm{~ns}$ & $0.777 \mathrm{~ns}$ & $0.101 \mathrm{~ns}$ & $0.082 \mathrm{~ns}$ \\
\hline
\end{tabular}

Los asteriscos indican el nivel de significancia de las diferencias entre las medias de los tratamientos $(* * *)=P \leq 0.001,(* *)=P \leq$ $0.01,\left({ }^{*}\right)=P \leq 0.05,(\mathrm{~ns})=\mathrm{P}>0.05$ (no significativo). 

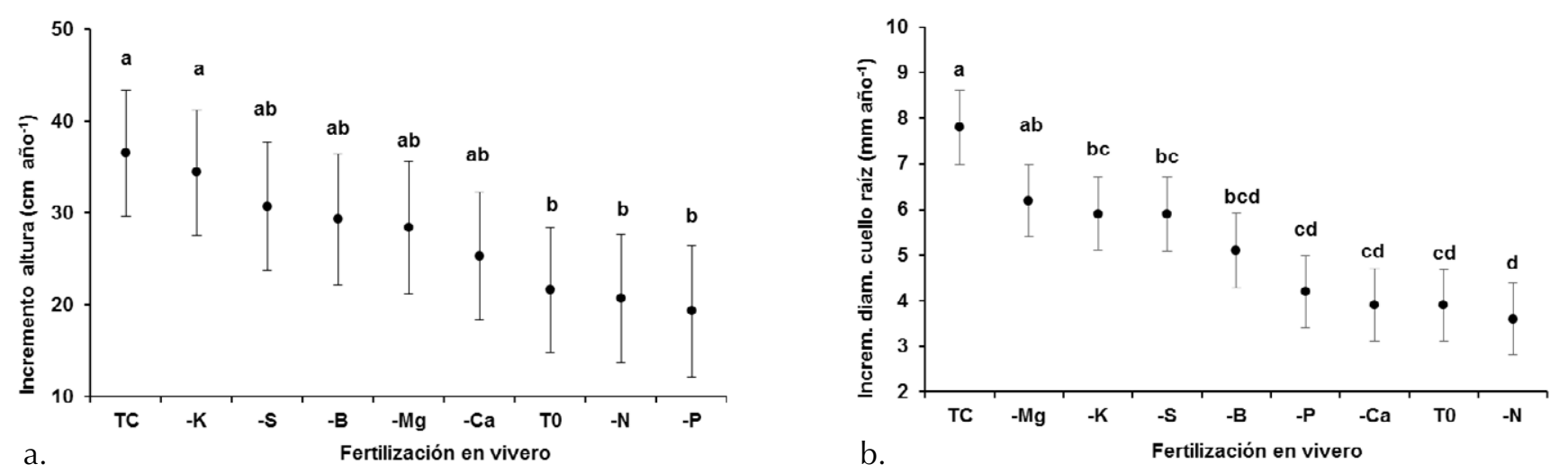

Figura 2. Efecto de la aplicación de diferentes tipos de fertilizante en vivero sobre a. el incremento anual en altura y b. el incremento anual en diámetro del cuello de la raíz, de árboles de Q. humboldtii después de un año de establecimiento en campo en cobertura de rastrojo alto. Los tipos de fertilizante aplicados en vivero fueron: TC = tratamiento completo, $-\mathrm{N}=$ tratamiento completo menos nitrógeno, $-\mathrm{P}=$ tratamiento completo menos fósforo, $-\mathrm{K}=$ tratamiento completo menos potasio, $-\mathrm{Ca}=$ tratamiento completo menos calcio, $-\mathrm{Mg}=$ tratamiento completo menos magnesio, $-\mathrm{S}=$ tratamiento completo menos azufre, $-\mathrm{B}=$ tratamiento completo menos boro, $\mathrm{T} 0=$ tratamiento testigo. Las barras en las figuras corresponden a intervalos de confianza de Tukey del $95 \%$.

fertilización sin nitrógeno (-N). La diferencia en el desarrollo promedio entre los árboles que más crecieron y los que menos crecieron fue de aproximadamente $19 \mathrm{~cm}$ año-1 en altura y $4 \mathrm{~mm}$ año ${ }^{-1}$ en diámetro del cuello de la raíz, las cuales equivalen a una diferencia cercana al $50 \%$ en el crecimiento en el campo entre unos y otros.

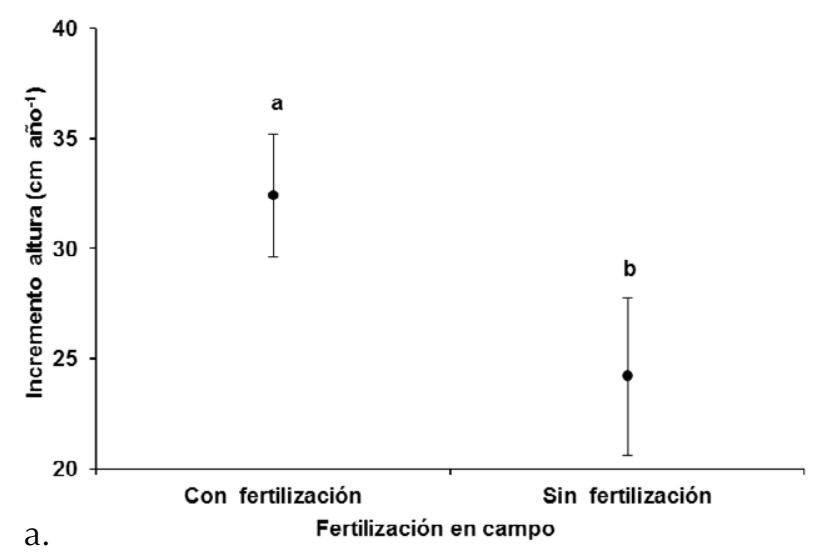

En el caso de la fertilización al momento de la plantación (FP), se evidenció un efecto positivo de esta práctica con respecto al incremento anual en altura y diámetro del cuello de la raíz de las plantas de roble andino (figura 3). La diferencia promedia entre los árboles fertilizados y no fertilizados en campo fue de aproximadamente 8 $\mathrm{cm}$ año $\mathrm{O}^{-1}$ en el incremento en altura y de $1.6 \mathrm{~mm}$

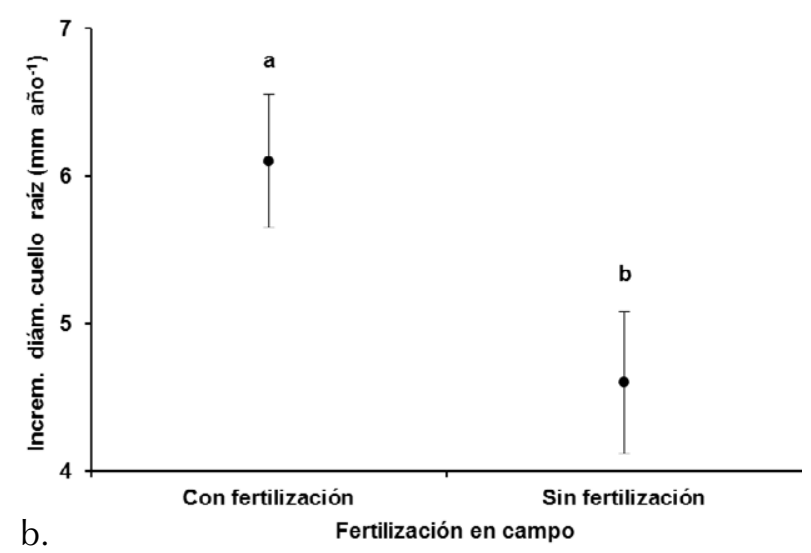

Figura 3. Efecto de la aplicación de fertilizante en campo al momento de la plantación sobre a. El incremento anual en altura y $\mathbf{b}$. El incremento anual en diámetro del cuello de la raíz de árboles de $Q$. humboldtii después de un año de plantación en cobertura de rastrojo alto. La fertilización aplicada fue de $35 \mathrm{~g}$ de 10:30:10 al fondo del hoyo a las plantas del tratamiento con fertilización. Las barras en las figuras corresponden a intervalos de confianza de Tukey del $95 \%$. 
año-1 en el incremento en el diámetro en el cuello de la raíz.

\section{Efecto de las condiciones en vivero y la fertilización en campo sobre el desarrollo de la plantación monoespecífica de roble establecida a plena exposición en cobertura de pasto}

El manejo en vivero y la fertilización en campo no tuvieron efecto significativo sobre la sobrevivencia de los árboles de roble en este sistema de plantación y la sobrevivencia promedio fue del $98.2 \%$. Pero la iluminación relativa en la que crecieron los árboles de roble en vivero sí tuvo un efecto significativo sobre el desarrollo de la plantación en esta cobertura, que se expresó en los valores del incremento en altura y diámetro del cuello de la raíz. En este último parámetro también se evidenció el efecto del tipo de fertilizante aplicado en vivero. La fertilización en campo al momento de la plantación y las interacciones no fueron significativas para los parámetros evaluados (tabla 3).

La evaluación particular del efecto de la iluminación relativa en vivero muestra que el ambiente lumínico afectó de igual manera los parámetros incremento anual en altura y diámetro en el cuello de la raíz. Los árboles que crecieron con alta iluminación relativa en vivero alcanzaron mayores valores de incremento para estos parámetros, los que disminuyeron en las plantas bajo menor iluminación (figura 4).

Tabla 3. Valores de $\mathrm{P}$ del efecto de los tratamientos sobre el desarrollo de árboles de roble andino (Q. humboldii) después de un año de su plantación en pastizales (efectos significativos en negrilla).

FC: fertilización en campo, IRV: iluminación relativa en vivero, FV: tipo de fertilizante aplicado en vivero.

\begin{tabular}{lccccccc}
\hline \multicolumn{1}{c}{ Variable } & FC & IRV & FV & FCxIRV & FCxFV & IRVxFV & FCxIRVxFV \\
\hline $\begin{array}{l}\text { Incremento anual altura } \\
\text { Incremento anual diámetro }\end{array}$ & $0.599 \mathrm{~ns}$ & $0.007^{* *}$ & $0.692 \mathrm{~ns}$ & $0.858 \mathrm{~ns}$ & $0.7509 \mathrm{~ns}$ & $0.292 \mathrm{~ns}$ & $0.925 \mathrm{~ns}$ \\
cuello de la raíz & $0.963 \mathrm{~ns}$ & $0.008^{* *}$ & $0.003^{* *}$ & $0.718 \mathrm{~ns}$ & $0.504 \mathrm{~ns}$ & $0.171 \mathrm{~ns}$ & $0.973 \mathrm{~ns}$ \\
\hline
\end{tabular}

Los asteriscos indican el nivel de significancia de las diferencias entre las medias de los tratamientos $(* * *)=P \leq 0.001,\left({ }^{* *}\right)=P \leq$ $0.01,(*)=P \leq 0.05,(\mathrm{~ns})=\mathrm{P}>0.05$ (no significativo).

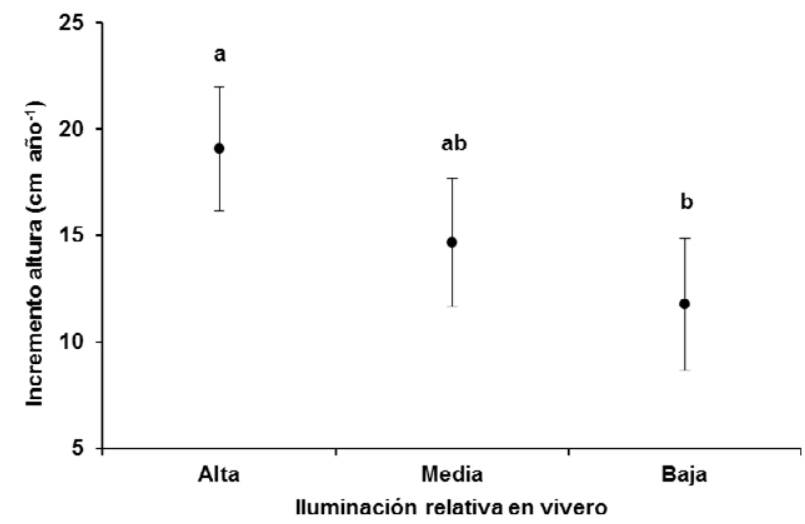

a.

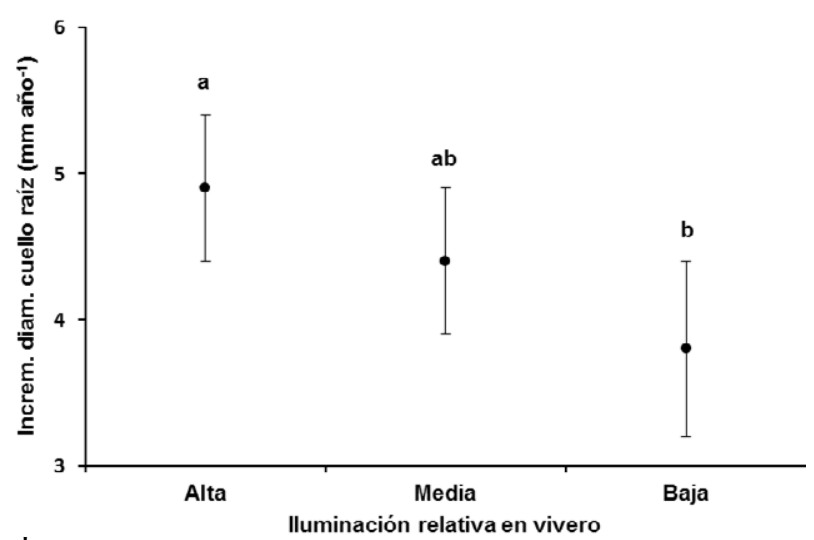

b.

Figura 4. Efecto de la iluminación relativa en vivero (alta: $100 \%$, media: $30 \%$ y baja: $15 \%$ ) sobre a. El incremento anual en altura y b. El incremento anual en diámetro del cuello de la raíz de árboles de $Q$. humboldtii después de un año de plantación en cobertura de pastizal. Las barras en las figuras corresponden a intervalos de confianza de Tukey del $95 \%$. 
De otro lado, el efecto del tipo de fertilizante que se utilizó en vivero sobre el incremento anual del cuello de la raíz (figura 5) muestra que el grupo de plantas con mayor crecimiento estuvo conformado por las que recibieron en vivero el fertilizante completo (TC), sin potasio (-K) y sin azufre (-S), mientras que el menor crecimiento se presentó en las que no recibieron fertilización (T0) y fertilizadas sin nitrógeno $(-\mathrm{N})$.

\section{Efecto del tipo de cobertura sobre el desarrollo de $\mathbf{Q}$. humboldtii}

El tipo de cobertura en el cual se establecieron los árboles determinó diferencias significativas sobre el incremento en altura y el diámetro del cuello de la raíz (tabla 4). Los árboles establecidos en la cobertura de rastrojo presentaron incrementos en altura superiores que los establecidos en la de pastos. La diferencia promedio fue de $12 \mathrm{~cm}$ por año aproximadamente $(82.4 \%$ por encima del crecimiento en los pastizales). Sin embargo, el crecimiento diamétrico del roble tuvo un comportamiento inverso pues los mayores valores se presentaron en los pastizales, aunque las diferencias fueron más modestas (el crecimiento de los árboles en los pastizales superó al de los árboles en los rastrojos solo en $1.5 \mathrm{~mm}$ año ${ }^{-1}$, una ventaja equivalente al $31.9 \%$ ). De otro lado, la mortalidad de los árboles establecidos en ambas coberturas fue

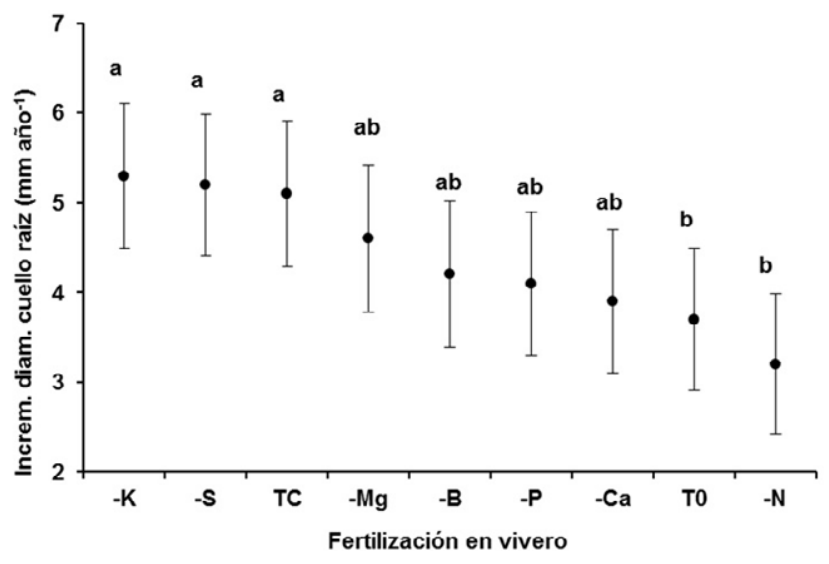

Figura 5. Efecto del tratamiento de fertilización en vivero sobre el incremento anual en diámetro del cuello de la raíz de árboles de $Q$. humboldtii después de un año de plantación en cobertura de pastizal.

$\mathrm{TC}=$ tratamiento completo, $-\mathrm{N}=$ tratamiento completo menos nitrógeno, $-\mathrm{P}=$ tratamiento completo menos fósforo, $-\mathrm{K}=$ tratamiento completo menos potasio, $-\mathrm{Ca}=$ tratamiento completo menos calcio, $-\mathrm{Mg}=$ tratamiento completo menos magnesio, $-\mathrm{S}=$ tratamiento completo menos azufre, $-\mathrm{B}=$ tratamiento completo menos boro, $\mathrm{T} 0=$ tratamiento testigo.

Tabla 4. Incremento anual en altura y diámetro del cuello de la raíz de árboles de Q. humboldtii establecidos a plena exposición en pastizales y bajo dosel de rastrojo alto.

La columna \% expresa las diferencias de cada variable en porcentaje con respecto a la cobertura de menor valor.

\begin{tabular}{lcccc}
\hline \multicolumn{1}{c}{ Tipo de plantación } & $\begin{array}{c}\text { Plantación a plena } \\
\text { exposición en pasto }\end{array}$ & $\begin{array}{c}\text { Plantación en } \\
\text { enriquecimiento de rastrojo }\end{array}$ & $\%$ & Valor $\boldsymbol{P}$ \\
\hline $\begin{array}{l}\text { Incremento en altura } \\
(\mathrm{cm} \text { año-1). }\end{array}$ & $15.3 \pm 1.9$ & $27.9 \pm 2.6$ & 82.4 & 0.0000 \\
$\begin{array}{l}\text { Incremento del diámetro de } \\
\text { cuello de la raíz }(\mathrm{mm} \text { año-1). }\end{array}$ & $6.2 \pm 0.5$ & $4.7 \pm 0.6$ & 31.9 & 0.0510 \\
\hline
\end{tabular}


casi nula, pues solo se presentó la muerte de tres individuos en la cobertura de pastos (lo que equivale al $1.8 \%$ ) y de uno en la cobertura de rastrojo (equivalente al $0.6 \%$ ).

\section{DISCUSIÓN}

De acuerdo con los resultados de este trabajo, el manejo en vivero y la fertilización al momento de la plantación no afectaron la sobrevivencia de los árboles de $Q$. humboldtii, pero sí su crecimiento en campo. No obstante, se espera que en el mediano plazo los árboles de menor vigor y crecimiento presenten mayor probabilidad de morir. En consecuencia, el manejo en vivero podría tener un efecto significativo sobre la sobrevivencia en el mediano y largo plazo, lo cual ha sido reportado en estudios realizado con otras especies de Quercus en diferentes países (Oliet et al., 2009; Villar-Salvador et al., 2013).

El tipo de factores que fueron determinantes sobre el crecimiento dependió del sistema de plantación utilizado. Los resultados contrastantes de crecimiento diamétrico y en altura entre las coberturas evaluadas sugieren que la especie presenta capacidad de aclimatación, entendida como el ajuste morfológico y fisiológico de los individuos en respuesta al estrés producido por cambios en las condiciones ambientales (Lambers et al., 2008). El marcado aumento del crecimiento en altura de los árboles establecidos bajo dosel de rastrojo alto abre una ventana de oportunidad excepcional para la plantación de esta especie en fajas de enriquecimiento en bosques secundarios degradados. Bajo tales condiciones, los troncos suelen ser rectos y poco ramificados. Por lo cual, estos resultados muestran el potencial de cultivar esta especie para producción de madera, tan apreciada para la industria licorera nacional.

De otro lado, los mayores crecimientos diamétricos y la baja mortalidad registrados en pastizales a plena exposición muestran el potencial de esta especie para proyectos de reforestación en zonas abiertas o pastizales degradados de la región andina, con fines comerciales o de conservación y restauración. Este resultado es de gran relevancia para los proyectos de restauración ecológica en las zonas altoandinas de Colombia, toda vez que el roble andino es la especie dominante de los bosques primarios en tales zonas, los cuales han sufrido fuerte destrucción para ser reemplazados principalmente por ganadería y agricultura.

El nivel de sombreamiento de los árboles en vivero no tuvo efecto sobre el crecimiento de los árboles de $Q$. humboldtii plantados bajo dosel de rastrojo. Bajo tal cobertura, los árboles presentaron una forma elongada con preponderancia del meristemo apical, conservaron las hojas que traían del vivero y se aclimataron bien a las condiciones de sombra intermedia del rastrojo, independientemente de la iluminación en la que habían crecido en el vivero. Por el contrario, los árboles en pastizales presentaron en general menor tamaño, en muchos casos debido a la muerte de la yema apical y el desarrollo de yemas laterales. El hecho de que los árboles provenientes de la condición de iluminación relativa alta en vivero fuesen los que mejor se desempeñaron en esta cobertura tiene que ver con que su condición en vivero fue la más parecida a la cobertura de pasto. A su vez, la mayoría de los árboles que provenían de los tratamientos de sombra media y baja en vivero presentaron defoliación casi completa en los primeros meses después del establecimiento en campo y, posteriormente, formaron hojas nuevas más pequeñas y de una tonalidad verde más clara. Este comportamiento indica que esta especie es capaz de aclimatarse a la sombra (Niinemets et al., 1998), pero es vulnerable a la fotoinhibición que ocurre cuando se transfiere a ambientes de alta iluminación (Osmond, 1994; Tognetti et al., 1997).

Estos resultados muestran que $Q$. humboldtii es una especie que en sus primeras etapas de desarrollo tolera y crece mejor en los ambientes de sombra intermedia, como se ha reportado en estudios de regeneración (Cabezas \& Ospina, 2010), ensayos de invernadero (Sepúlveda et al., 2014; 
Moreno \& Díez, 2008) y plantaciones en campo (Cabezas et al., 2008; Moreno \& Cuartas, 2015). No obstante, una vez los árboles mudaron las hojas de sombra fueron capaces de desarrollar nuevo follaje adaptado a la alta radiación, lo cual indica que el roble andino también puede sobrevivir y crecer, aunque más lentamente (aproximadamente por debajo de $25 \mathrm{~cm}$ por año en las etapas iniciales), en ambientes de alta iluminación como es el caso de la plantación en pastos.

Algunos estudios recientes de restauración de ecosistemas en México comparan la sobrevivencia y el crecimiento de especies neotropicales de Quercus tolerantes a la sombra ( $Q$. xalapensis y $Q$. germana) en pastos abandonados y bosques secundarios. Se reportan resultados diferentes a los de este estudio pues, aunque la sobrevivencia fue mayor en bosque secundario, la tasa de crecimiento relativo en altura fue superior en pastos abandonados. Esta diferencia se debe posiblemente a que se trataba de un bosque de niebla donde la nubosidad frecuente disminuye los factores de estrés por alta radiación en los pastizales abandonados y al alto sombreamiento de las plantas en el bosque secundario donde se plantaron (Muñiz-Castro et al., 2015), comparado con los ambientes en los que se plantó el $Q$. humboldtii en el presente trabajo.

Se ha reportado que $Q$. humboldtii se adapta a diferentes tipos de suelos, desde moderadamente fértiles y profundos hasta suelos en proceso de degradación (López, 2014), posiblemente debido a la capacidad de formar asociaciones ectomicorrizas que son eficientes en la adquisición de agua, fósforo y otros nutrientes (Zárate, 2011; Becerra \& Zack, 2011). Sin embargo, se pudo observar que las carencias generadas desde el vivero por la aplicación de fertilizantes con algún elemento faltante disminuyen considerablemente el crecimiento de las plantas de Q. humboldtii en campo, principalmente en el caso de las deficiencias de nitrógeno, fósforo y calcio en ambos tipos de plantación, lo cual podría implicar que la fertilización en vivero se restringiera a la aplicación de estos elementos.
Se ha reportado que los recursos maternos suministrados a través de las semillas grandes en otras especies de Quercus afectan el desarrollo de las plántulas (Quero et al., 2007). Pues el roble andino también tiene semillas grandes, se esperaría que este factor también fuera influyente. Sin embargo, los efectos de la fertilización en vivero sobre el crecimiento en el campo reportados en este estudio y en un trabajo previo en la fase de vivero (Sepúlveda et al., 2014) sugieren que el efecto de las reservas maternas sobre el comportamiento de las plantas (tanto en vivero como en campo) fue despreciable. No obstante, por su importancia, este es un factor que debería estudiarse con mayor profundidad en investigaciones futuras sobre esta especie.

La aplicación del fertilizante NPK al momento de la plantación tuvo efecto sobre el crecimiento de Q. humboldtii solamente cuando se plantaron bajo rastrojo, pero no en pastos. En otras especies del bosque andino en Colombia (Baccharis macrantha y Viburnum triphyllum) se ha reportado una situación similar, pues cuando se plantaron en pastizales la aplicación de nutrientes al momento de la plantación no tuvo efecto sobre el crecimiento inicial de las plantas (Hernández-Pineda et al., 2014). Esto puede deberse a varias razones. En primer lugar, la historia de uso de estos pastizales abandonados, los cuales generalmente se han utilizado en cultivos anuales que se fertilizan regularmente o como pastos manejados donde es común la aplicación de urea, por lo cual los niveles de concentración de nutrientes en el suelo pueden ser suficientes para el desarrollo de los árboles de roble y la fertilización adicional no incrementa su desarrollo. La falta de respuesta a la aplicación de fertilizantes en los robles plantados a plena exposición en pastizales también puede deberse a que el estrés producido por alta radiación limita el crecimiento y, por tanto, anula el efecto de la fertilización. Futuras investigaciones dirigidas a comprender mejor la autoecología y manejo de esta especie tan promisoria de las montañas de 
Colombia deberán abordar estas interacciones en mayor profundidad.

\section{CONCLUSIONES}

La sobrevivencia de los árboles jóvenes de roble no fue afectada por las condiciones de manejo en vivero (IR y fertilización), ni por las condiciones de cobertura y fertilización en campo.

El crecimiento en altura de árboles jóvenes de roble, luego del establecimiento en campo, fue favorecido por la cobertura vegetal de rastrojo; mientras que el crecimiento diamétrico se favoreció a plena exposición. No obstante, aparentemente el rastrojo genera condiciones más favorables para el crecimiento del roble.

Bajo la cobertura de rastrojo la fertilización tanto en vivero como en campo mejoró el crecimiento de los árboles jóvenes de roble. Las deficiencias nutricionales heredadas desde la fase de vivero restringieron el crecimiento de los árboles una vez fueron llevados a campo. Las deficiencias en $\mathrm{N}, \mathrm{P}$ y Ca fueron más restrictivas que las de B, $\mathrm{Mg}, \mathrm{S}$ y $\mathrm{K}$.

Bajo la cobertura de pastos la fertilización tanto en vivero como en campo no mejoró el crecimiento en altura de los árboles jóvenes de roble. En contraste, la fertilización en vivero sí afectó significativamente el incremento en diámetro en el cuello de la raíz al final del periodo de monitoreo en campo. En este caso la deficiencia en $\mathrm{N}$ fue la más restrictiva, mientras que las deficiencias en $\mathrm{S}$ y $\mathrm{K}$ fueron las menos restrictivas. Así mismo, los árboles que crecieron en condición de iluminación relativa alta en vivero exhibieron un mejor crecimiento en altura y diámetro en el cuello de la raíz cuando se plantaron en cobertura. Al disminuir la iluminación relativa a la cual estuvieron expuestos los árboles en vivero, se notó una disminución en el crecimiento en altura y diámetro del cuello de la raíz de los árboles jóvenes en campo.

\section{AGRADECIMIENTOS}

Este trabajo se realizó como parte de las actividades del proyecto "Ecología y silvicultura del roble de tierra fría (Quercus humboldtii Bonpl.)", cofinanciado por la Corporación Autónoma Regional para el Centro de Antioquia (Corantioquia), la Universidad Nacional de Colombia y el programa Jóvenes investigadores de Colciencias.

\section{CONFLICTO DE INTERESES}

El autor declara no tener conflicto de intereses.

\section{CONTRIBUCIÓN POR AUTOR}

El autor único es responsable de la obra en todos los aspectos que condujeron a la elaboración de su publicación.

\section{REFERENCIAS BIBLIOGRÁFICAS}

Andivia, E., Carevic, F., Fernández, M., Alejano, R., Vázquez-Piqué, J. \& Tapias, R. (2012). Seasonal evolution of water status after outplanting of two provenances of Holm oak nursery seedlings. New Forests, 43(5-6), 815-824.

Armenteras, D., Rodríguez, N., Retana, J. \&Morales, M. (2011). Understanding deforestation in montane and lowland forests of the Colombian Andes. Regional Environmental Change, 11(3),693-705.

Becerra, A. G. \& Zak, M. R. (2011). The ectomycorrhizal symbiosis in South America: morphology, colonization, and diversity. En M. Rai \& A. Varma (eds.). Diversity and biotechnology of ectomycorrhizae (pp. 19-41). Berlin: Springer-Verlag.

Cabezas, A. \& Ospina, R. (2010). Análisis del paisaje y de su relación con la regeneración del roble (Quercus humboldtii Bonpl.) en el municipio de Popayán, departamento del Cauca. Colombia Forestal, 13(2), 189-200. 
Cabezas, M., Peña, F., Díaz, C. J. \& Moreno, A. F. (2008). Dosel de tres especies forestales y su relación con la adaptación a suelos degradados por la erosión. Revista UDCA Actualidad \& Divulgación Científica, 11(2), 175-185.

Cárdenas, D. \& Salinas, N. (2006). Roble: Quercus humboldtii Bonpl. En D. Cárdenas \& N. R. Salinas (eds.). Libro rojo de plantas de Colombia. Especies maderables amenazadas: primera parte (pp. 156160). Bogotá: Instituto Amazónico de Investigaciones Científicas (Sinchi).

Cervantes, V., López, M., Salas, N. \& Hernández, G. (2001). Técnicas para propagar especies nativas de la selva baja caducifolia y criterios para establecer áreas de reforestación. México D.F: Facultad de Ciencias, Universidad Nacional Autónoma de México. $52 \mathrm{p}$.

Empresas Públicas de Medellín. (1994). Boletín hidrometeorológico. Volumen 1-2. Medellín: Empresas Públicas de Medellín. 80 p.

Gilman, E.F. (1994). Establishing trees in the landscape. En D. Neely, \& G.W.Watson (eds.). The Landscape Below Ground (pp. 69-77). Proceedings of an International Workshop on Tree Root Development in Urban Soils. Champaign (IL): International Society of Arboriculture.

Hernández-Pineda, L. L., Roa-Casas, O. M. \& Cortés-Pérez, F. (2014). Crecimiento de Baccharis macrantha y Viburnum triphyllum, dos especies nativas útiles en restauración ecológica, plantadas en un pastizal andino (Boyacá, Colombia). Biota Colombiana, 15, 27-38.

Holdridge, L. R. 1996. Ecología basada en las zonas de vida. San José (Costa Rica): Instituto Interamericano de Ciencias Agrícolas. 216 p.

Lambers, H., Chapin III, F. S. \& Pons, T. L. (2008). Plant Physiological Ecology. Second Edition. New York: Springer-Verlag. 604 p.

Landis, T.D. (1985). Mineral nutrition as an index of seedling quality. En: M.L. Duryea (ed.). Evaluating seedling quality: Principles, procedures, and predictive abilities of major tests (pp. 29-48). Corvallis: Forest Research Laboratory, Oregon State University.
López, D. C. (2014). Predicción de la futura distribución potencial de Quercus humboldtii bajo diferentes escenarios de cambio climático (Tesis M.Sc. Sistemas de Información Geográfica). Quito: Universidad San Francisco de Quito. 70 p.

Manos, P. S., Doyle, J. J. \& Nixon, K. C. (1999). Phylogeny, biogeography, and processes of molecular differentiation in Quercus subgenus Quercus (Fagaceae). Molecular Phylogenetics and Evolution, 12(3), 333-349.

Moreno, D. J. \& Cuartas, S. E. (2015). Survival and growth of tree seedlings from three species in degraded andean mountain forest for cattle grazing in Colombia. Acta Biológica Colombiana, 20(2), 85-100.

Moreno, F. H. \& Díez, M.C. (2008). Crecimiento de 11 especies arbóreas de los bosques altoandinos en condiciones contrastantes de luz. En J. D. León (ed.). Ecología de bosques andinos. Experiencias de investigación (pp. 59-77). Medellín: La Carreta Editores E.U.

Muñiz-Castro, M. A., Williams-Linera, G. \& BenítezMalvido, J. (2015). Restoring montane cloud forest: establishment of three Fagaceae species in the old fields of central Veracruz, Mexico. Restoration Ecology, 23(1), 26-33.

Myers, N., Mittermeier, R. A., Mittermeier, C. G., Da Fonseca, G. A. \& Kent, J. (2000). Biodiversity hotspots for conservation priorities. Nature, 403(6772), 853-858.

Niinemets, U., Kull, O. \& Tenhunen, J.D. (1998). An analysis of light effects on foliar morphology, physiology, and light interception in temperate deciduous woody species of contrasting shade tolerance. Tree Physiology, 18, 681-696

Nixon, K. C. (1993). Infrageneric classification of Quercus (Fagaceae) and typification of sectional |names. Annales des Sciences Forestières, 50, 25-34.

Oliet, J. A., Tejada, M., Salifu, K. F., Collazos, A. \& Jacobs, D. F. (2009). Performance and nutrient dynamics of holm| oak (Quercus ilex L.) seedlings in relation to nursery nutrient loading and post-transplant fertility. European Journal of Forest Research, 128(3), 253-263. 
Osmond, C.B. (1994). What is photoinhibition? Some insights from comparisons of shade and sun plants. En: N.R. Baker \& J.R. Bowyer (eds.). Photoinhibition of photosynthesis: from molecular mechanisms to the field (pp. 1-24). Oxford: BIOS Scientific Publishers Limited.

Pacheco, R. A. \& Pinzon, C. A. (1997). El Roble Quercus humboldtti Bonpl. Bogotá: Jardín botánico de Bogotá José Celestino Mutis.

Quero, J. L., Villar, R., Marañón, T., Zamora, R. \& Poorter L. (2007). Seed-mass effects in four Mediterranean Quercus species (Fagaceae) growing in contrasting light environments. American Journal of Botany, 94,1795-1803

Sepúlveda, Y. L., Díez, M. C., Moreno, F. H., León, J. D. \& Osorio, N. W. (2014). Efectos de la iluminación relativa y la fertilización sobre el crecimiento de plántulas de roble andino en vivero. Acta Biológica Colombiana, 19(2), 211-220.

Tejedor, N., Álvarez, E., Arango, S., Araujo, A., Blundo, C., Boza, T., La Torre, M.A., Gaviria, J., Gutiérrez, N., Jørgensen, P.M., León, B., López, R., Malizia, L., Millán, B., Moraes, M. Pacheco, S., Rey, J.M., Reynel, C., Timaná, M., Ulloa, C., Vacas, O., Newton, A.C. (2012). Evaluación del estado de conservación de los bosques montanos en los Andes tropicales. Ecosistemas, 21(1-2), 148-166.

Tognetti, R., Johnson, J. D. \& Michelozzi, M. (1997). Ecophysiological responses of Fagus sylvatica seedlings to changing light conditions. I. Interactions between photosynthetic acclimation and photoinhibition during simulated canopy gap formation. Physiologia Plantarum, 101(1), 115-123.

Trubat, R., Cortina, J. \& Vilagrosa, A. (2011). Nutrient deprivation improves field performance of woody seedlings in a degraded semi-arid shrubland. ECOlogical Engineering, 37(8), 1164-1173.

van den Driessche, R. (1984). Relationship between spacing and nitrogen fertilization of seedlings in the nursery, seedling mineral nutrition and outplanting performance. Canadian Journal of Forest Research, 14, 431-436.

Villar-Salvador, P., Peñuelas, J. L., Nicolás-Peragón, J. L., Benito, L. F. \& Domínguez-Lerena, S. (2013). Is nitrogen fertilization in the nursery a suitable tool for enhancing the performance of Mediterranean oak plantations? New forests, 44(5), 733-751.

Villar-Salvador, P., Planelles, R., Enriquez, E. \& Rubira, J. P. (2004). Nursery cultivation regimes, plant functional attributes, and field performance relationships in the Mediterranean oak Quercus ilex L. Forest Ecology and Management, 196(2), 257-266.

Westerman, R. L., Baird, J. V. \& Christensen, N. W. (eds.). (1990). Soil testing and plant analysis. Madison: Soil Science Society of America Inc. (SSSA Book Series, 3). 760 p.

Zárate, L. M. (2011). Rendimiento de Quercus humboldtii Bonpland (roble) en condiciones de interacción micorrizas ectotróficas-humus. Acta Biológica Colombiana, 7(2), 64.

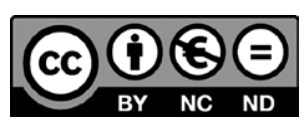

